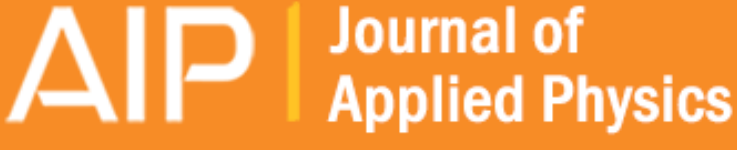

\section{Modeling of stochastic motion of bacteria propelled spherical microbeads}

Veaceslav Arabagi, Bahareh Behkam, Eugene Cheung, and Metin Sitti

Citation: Journal of Applied Physics 109, 114702 (2011); doi: 10.1063/1.3592970

View online: http://dx.doi.org/10.1063/1.3592970

View Table of Contents: http://scitation.aip.org/content/aip/journal/jap/109/11?ver=pdfcov

Published by the AIP Publishing

\section{Articles you may be interested in}

Magnetic resonant wireless power transfer for propulsion of implantable micro-robot

J. Appl. Phys. 117, 17E712 (2015); 10.1063/1.4918963

Biomanufacturing and self-propulsion dynamics of nanoscale bacteria-enabled autonomous delivery systems Appl. Phys. Lett. 105, 173702 (2014); 10.1063/1.4900641

Near and far-wall effects on the three-dimensional motion of bacteria-driven microbeads Appl. Phys. Lett. 102, 143701 (2013); 10.1063/1.4801810

Construction and operation of a microrobot based on magnetotactic bacteria in a microfluidic chip Biomicrofluidics 6, 024107 (2012); 10.1063/1.3702444

Bacterial flagella-based propulsion and on/off motion control of microscale objects Appl. Phys. Lett. 90, 023902 (2007); 10.1063/1.2431454

Frustrated by

old technology?

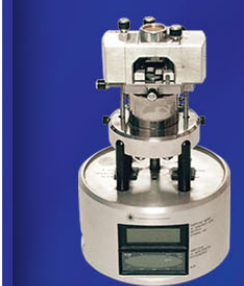

Is your AFM dead and can't be repaired?

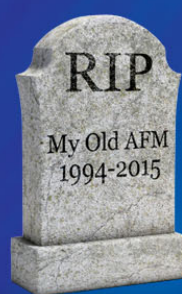

Sick of bad customer support?

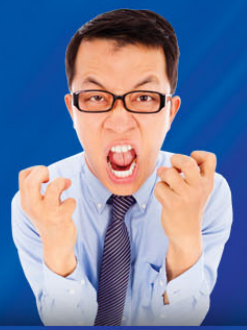

It is time to upgrade your AFM Minimum $\$ 20,000$ trade-in discount for purchases before August 31st

Asylum Research is today's technology leader in AFM 


\title{
Modeling of stochastic motion of bacteria propelled spherical microbeads
}

\author{
Veaceslav Arabagi, ${ }^{1, a)}$ Bahareh Behkam, ${ }^{2, a)}$ Eugene Cheung, ${ }^{1}$ and Metin Sitti ${ }^{1, b}$ \\ ${ }^{1}$ Department of Mechanical Engineering, Carnegie Mellon University, Pittsburgh, Pennsylvania 15213, USA \\ ${ }^{2}$ Department of Mechanical Engineering, Virginia Tech, Blacksburg, Virginia 24061, USA
}

(Received 14 February 2011; accepted 21 April 2011; published online 6 June 2011)

\begin{abstract}
This work proposes a stochastic dynamic model of bacteria propelled spherical microbeads as potential swimming microrobotic bodies. Small numbers of $S$. marcescens bacteria are attached with their bodies to surfaces of spherical microbeads. Average-behavior stochastic models that are normally adopted when studying such biological systems are generally not effective for cases in which a small number of agents are interacting in a complex manner, hence a stochastic model is proposed to simulate the behavior of 8-41 bacteria assembled on a curved surface. Flexibility of the flagellar hook is studied via comparing simulated and experimental results for scenarios of increasing bead size and the number of attached bacteria on a bead. Although requiring more experimental data to yield an exact, certain flagellar hook stiffness value, the examined results favor a stiffer flagella. The stochastic model is intended to be used as a design and simulation tool for future potential targeted drug delivery and disease diagnosis applications of bacteria propelled microrobots. (C) 2011 American Institute of Physics. [doi:10.1063/1.3592970]
\end{abstract}

\section{INTRODUCTION}

Miniature mobile robots have several useful and unique abilities such as accessing small spaces and the capability of parallel operation with large numbers of agents via distributed control. These characteristics render them indispensable for mobile sensor network applications for environmental monitoring, search and rescue, homeland security, surveillance, and health-care applications for minimally invasive diagnosis and treatment inside or outside the human body, among many others. However, current miniature robots are limited to centimeter or millimeter scale overall sizes primarily due to the miniaturization limits of their on-board actuators and power sources. ${ }^{1}$

To solve the grand challenge of actuation of micron scale mobile robots, two general approaches have been proposed in literature. The first approach, based on off-board actuation and powering, enabled the development of nontethered microrobots, among which are, an electrostatic scratch drive silicon robot $^{2}$ and external magnetic field actuated robots. ${ }^{3,4}$ Furthermore, magnetic field gradients have been used to actuate bioinspired structures inspired by both eukaryotic and prokaryotic flagella as another microscale swimming propulsion demonstration. ${ }^{5,6}$ These off-board powering and actuation methods work only in limited workspaces and require expensive and bulky external instrumentation.

The second approach utilizes biological motors that are isolated from microorganisms and used as new micro/nanoscale actuators. Soong et al. ${ }^{7}$ used $F_{1}$-ATPase rotary biological motors to rotate hundreds of chemically attached nanometer-size nickel rods at around $8 \mathrm{~Hz}$ speeds. These bio-

\footnotetext{
a) Electronic mail: varabagi@andrew.cmu.edu; behkam@vt.edu.

b) Author to whom correspondence should be addressed. Electronic mail: sitti@cmu.edu.
}

motors are fueled by adenosine triphosphate (ATP) in a liquid environment. More recently, flagellated bacteria have been utilized as actuators in liquid media. Flagellum is the propulsive organelle of bacteria composed from a reversible rotary motor embedded in the cell wall and a filament that extends into the external medium. ${ }^{8}$ Flagellar motors of bacteria rotate due to proton motive force or in some cases a sodium ion gradient. $^{9-11}$ The flagellar filament is a long (around $10 \mu \mathrm{m}$ ) and thin (around $20 \mathrm{~nm})$ cylindrical helix $(2.5 \mu \mathrm{m}$ pitch and 0.5 $\mu$ m diameter) that rotates at speeds of up to $100 \mathrm{~Hz} .{ }^{12}$ Hydrodynamic interactions among flagella cause them to coordinate, coalesce and bundle behind the cell during swimming. ${ }^{13}$ Flagellated bacteria have been employed by multiple research groups for a variety of different applications. Darnton et al. ${ }^{13}$ accomplished active mixing by adsorbing swarmer cells of Serratia marcescens (S. marcescens) to polydimethylsiloxane (PDMS) surfaces. They also demonstrated that these flagellated bacteria can adhere to microscale objects, such as polystyrene beads and PDMS pieces, and propel them. Kim et $a l .{ }^{14,15}$ also showed that surface arrays of $S$. marcescens can be utilized to achieve mixing in microfluidic channels and ultra violet light controlled propulsion of PDMS "barges" propelled by the same bacteria. Tung et al. ${ }^{16}$ developed a microfluidic pump by tethering a linear array of smooth swimming Escherichia coli (E. coli) KAF95 along the sidewall of a microchannel. Magnetotactic bacteria MC-1, which can move at speeds up to $100 \mu \mathrm{m} / \mathrm{s}$, have also been used for propulsion of microscale beads. ${ }^{17}$ Swarms of these bacteria have been employed for controlled propulsion of microfabricated objects, several hundreds of microns in size, however these bacteria grow in anaerobic or microaerobic conditions, which could limit their application. ${ }^{18}$ Behkam and Sitti have shown that $S$. marcescens bacteria can be used to propel microrobots of various geometry and sizes in a static fluid. ${ }^{19,20}$ The authors also introduced a chemical switching method for control of 
the on/off motion of the bacteria-propelled objects. Additionally, they have extensively studied the adhesion of bacteria to various micro-objects and proposed chemical and physical patterning techniques for controlled adhesion of bacteria to surfaces, in order to achieve higher speeds and more directional motion trajectories. ${ }^{21,22}$

Due to the stochastic nature of bacteria motion, control of the trajectory of bacteria-propelled objects is not a trivial task. Statistical based approaches and modeling have been proposed in, ${ }^{23}$ however these approaches are based to a large extent on the mean behavior of a large population of bacteria propelling planar geometry. Collective behavior models may not be suitable for modeling systems in which the number of agents (i.e., bacteria) are reduced to a few tens and a more complex and nonplanar three-dimensional (3D) geometry is introduced. In such cases, each bacterium's stochastic behavior influences the overall motion of the system and the simple average behavior models may not be able to predict the overall system behavior accurately.

In this work, a theoretical model for the stochastic motion of nonplanar 3D geometries propelled by a small number of bacteria is introduced. It is shown that this noncollective behavior stochastic model is able to predict to a certain extent the motion of objects with curved geometry $(10 \mu \mathrm{m}$ diameter beads) with a low number of attached bacteria $(8-41)$. Spherical microbeads are selected as the 3D objects since they are commercially available from a wide range of polymer materials, available with various coatings. Simulation results are compared with previously reported experimental results for propulsion of $10 \mu \mathrm{m}$ polystyrene beads by an ensemble of S. marcescens bacteria. ${ }^{19,21}$ Due to the intrinsic stochasticity of bead motion, it is impossible to duplicate the exact experimental trajectories of the beads, hence emergent parameters of bead mean squared displacement and velocity are employed to validate the undertaken modeling approach. Furthermore, the theoretical simulation is used to reveal insight into the attachment of bacteria on the surface of the bead, the compliance of the flagellar hook, as well as details of propulsive force generation in flagellar motors.

The paper is organized as follows. In Sec. II, the theoretical model serving as the basis for the simulation is presented along with details on bacterial attachment, the propulsive mechanism, and bead dynamics. To model the degree of conformation of the flagella bundle with the surrounding fluid flow, the notion of "flexibility of the flagellar hook" is proposed, as well as, the fluid flow in the vicinity of the bead is modeled under Stokes flow regime. In Sec. III, the effect of flagellar hook flexibility is examined through several simulated scenarios. Comparison to experimental results favors a somewhat rigid flagellar hook, although, since experimental data is limited, the exact value of hook stiffness cannot be determined. Finally, the concept of randomization time $\left(\tau_{R}\right)$ is discussed and the propulsion of beads at long $\left(\Delta t \gg \tau_{R}\right)$ timescales is examined to capture the random walk behavior of the bead.

\section{MODELING}

Due to the low number of bacteria propelling the bead, each bacterium's individual stochastic behavior becomes vital to the overall trajectory. Hence, the theoretical model is based on modeling each bacterium's force and torque output and then computing its effect on the translational and rotational motion of the bead it is attached to. The location of attachment of bacteria onto the bead is also of primary importance as the point of propulsive force application greatly influences the dynamics of the bead. Furthermore, the orientation of the flagella affects the direction of the propulsion force and can significantly influence bead dynamics. A model for the flexible hook at the base of the flagella is developed to allow alignment of the flagella with the fluid flow field. This model is evaluated in light of the simulated and experimental results.

\section{A. Bacteria behavioral aspects}

Motile bacteria behavior is composed of two distinct states: run and tumble. The run state is characterized by a bacterium bundling its multiple flagella together and rotating them counterclockwise (CCW) to achieve propulsion in the forward direction, while during the tumble state one or more flagellar motor rotate clockwise $(\mathrm{CW})$ and the flagella unbundle ceasing to generate any propulsive force thereby allowing momentary Brownian diffusion only. These two operating states can be modeled as a continuous time Markov chain where state transitions occur in a Poisson manner. ${ }^{24}$ Hence, the transition probabilities between states, $\lambda_{r}$ and $\lambda_{t}$, can also be viewed as the arrival rates of the next state, explicitly $\lambda_{r}$ arrival rate of a run state when the bacteria is tumbling, $\lambda_{t}$ arrival rate of a tumble state when running. In absence of chemical stimuli in the medium, the run and tumble phases occur stochastically and, in combination with diffusion, result in the bacteria and bacteria-propelled objects performing a "random walk." Note that no chemotactic behavior is presented in this work, although the developed model can be easily extended to encompass it.

In general, this modeling approach is based on modeling each bacterium's transitions between its running and tumbling states, afterward propagating the produced resultant forces and moments into motion of the corresponding bead. Since the inter-arrival times between events are exponentially distributed in a Poisson process, they are described in the following manner:

$$
f(t, \lambda)=\lambda e^{-\lambda t}
$$

where $t$ is the time of an arrival event and $\lambda$ is the arrival rate of events. For the two-state Markov process describing bacterial state transitions, the "holding" or "dwell" time in each state can take values of $1 / \lambda_{r}=0.1 \mathrm{~s}$ for the tumbling state and $1 / \lambda_{t}=0.9 \mathrm{~s}$ for the running state. ${ }^{25}$ Due to the independence property of exponentially distributed arrival events, for each attached bacterium at each time step $(d t)$, a "next arrival" time is sampled from this distribution, via the inverse transform sampling technique, and decided whether the given bacterium will undergo a transition to running/tumbling in the next time step. This sampling is performed within a larger framework of an integration running forward in time. 


\section{B. Bacteria attachment}

Due to the small number of bacteria attached to the spherical bead, the orientation and location of each bacterium on the surface is of primary importance. In order to be able to validate theoretical findings from this model against experimental results previously obtained in, ${ }^{21}$ two bacteria attachment scenarios namely, "unpatterned" and "patterned" beads, are modeled. The patterned beads, named so because half of their surface was treated with air plasma, favor bacteria attachment to the treated hemisphere only, while the unpatterned beads have the bacteria attached over their entire surface (as shown in Fig. 1). In order to define the initial configuration of bacteria attached to a bead, the following heuristics and assumptions were imposed:

- Bacteria can only attach to the surface of the bead with their bodies. Attachment by flagella was rarely observed in experiments and did not last very long when it occurred.

- The attachment points of bacteria onto the surface of the bead are defined differently for patterned and unpatterned beads:

\section{Unpatterned beads}

Bacteria attachment points are uniformly distributed over the surface area of the spherical bead with an attachment density of 1 bacterium per $7 \mu \mathrm{m}^{2}$. This attachment density was consistently observed in experiments for adhesion of $S$. marcescens bacteria to pure polystyrene beads. ${ }^{21}$

\section{Patterned beads}

Bacteria attachment points are configured in a close packed hexagonal configuration. This assumption stems from experimental observations that even a small number of bacteria will cluster together. The density of attachment is taken to be 1 bacterium per $12 \mu \mathrm{m}^{2}$ based on experimental observations.

- Bacteria orientation is randomized within a bounded volume around the point of attachment to the sphere as portrayed in Fig. 2. The random original orientation of the bacteria stems from the fact that bacteria attach to the bead in static fluid, therefore no particular direction is preferred.

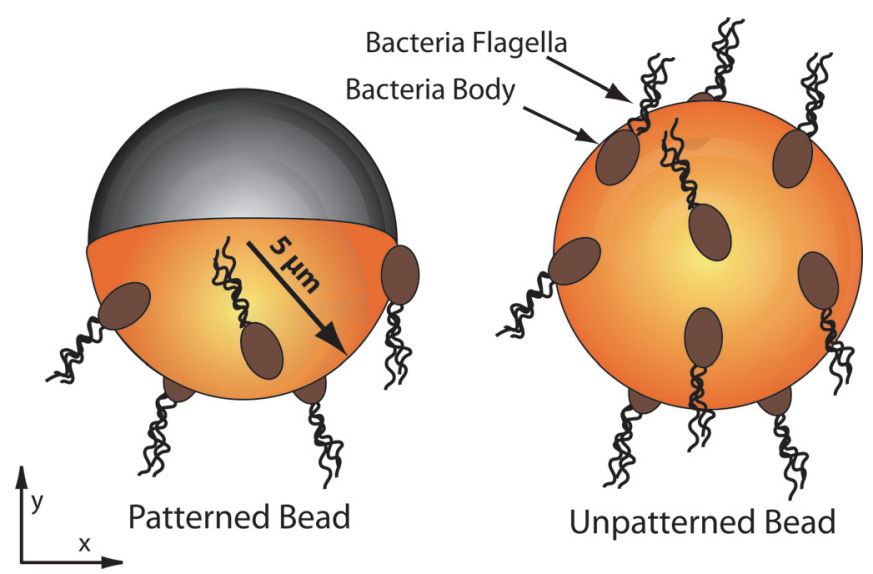

FIG. 1. (Color online) Schematic of attached bacteria configuration on patterned and unpatterned beads.
Furthermore, the random attachment angle of each bacteria is bounded between $\left[5^{\circ}-60^{\circ}\right]$, assumption that stems from consistent experimental observations that bacteria attach longitudinally (along their side) to the surface of the bead, while head-on attachment is very short lived, if present at all, suggesting its instability.

\section{Modeling flagellar hook flexibility}

A flagellum consists of three structural parts: the flagellar filament, the hook, and the basal structure. The flagellar filament is a tubular polymer structure which is capable of assuming several well-defined helical morphologies. The flagellar hook is a tubular structure which functions as a universal joint and plays the important role of enabling the flagella to function effectively as a bundle in peritrichous bacteria. The basal structure consists of several multicomponent complexes and is the main structural body of the flagellar motor. ${ }^{26}$

Accordingly, the bacterial flagellum is modeled as being attached to the body by an omni-directional torsional spring, allowing the flagella bundle to deflect when affected by the fluid flow in the bead's vicinity, as portrayed in Fig. 3. This assumption stems from the fact that experimental measurements suggest orders of magnitude larger stiffness for the flagella compared to the hook. ${ }^{27,28}$ To the best of our knowledge, torsional stiffness of the flexible hook for S. marcesecens bacteria has not been experimentally measured; hence, the stiffness of the hook is taken to be variable, thereby changing the sensitivity of the flagella to the surrounding flow, from being very stiff, not affected by fluid flow, to very flexible, fully conforming to the streamlines nearby the bead. Considerations of the fluid streamlines in the vicinity of the bead as well as a metric for hook flexibility are developed and implemented in the numerical model as presented below.

\section{Flagellar hook deflection modeling}

Since utilizing a metric for the actual torsional stiffness of the flexible hook presents no intuitive meaning and

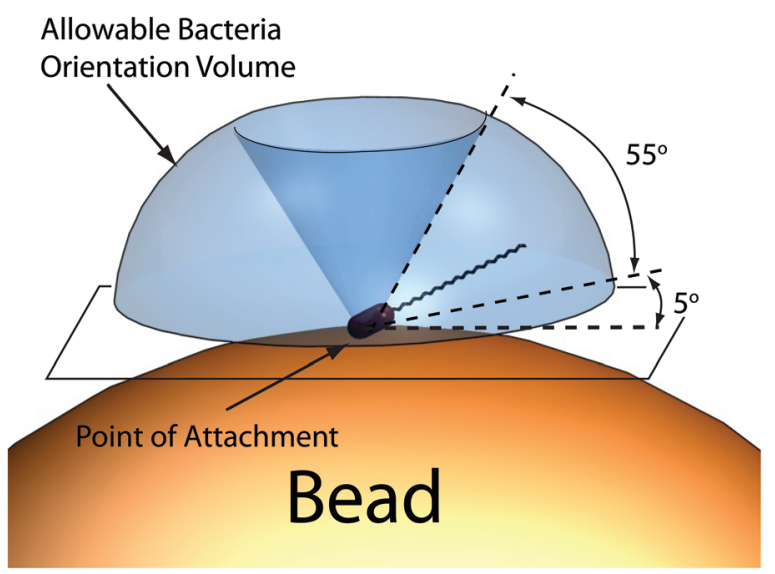

FIG. 2. (Color online) Bacteria attachment orientation: Given a certain bacterium attachment point to the surface of the bead, the flagella are given a random orientation that lies in the interval $\left[5^{\circ}-60^{\circ}\right]$ from the plane tangential to the bead, represented by the revolved volume in the figure. 


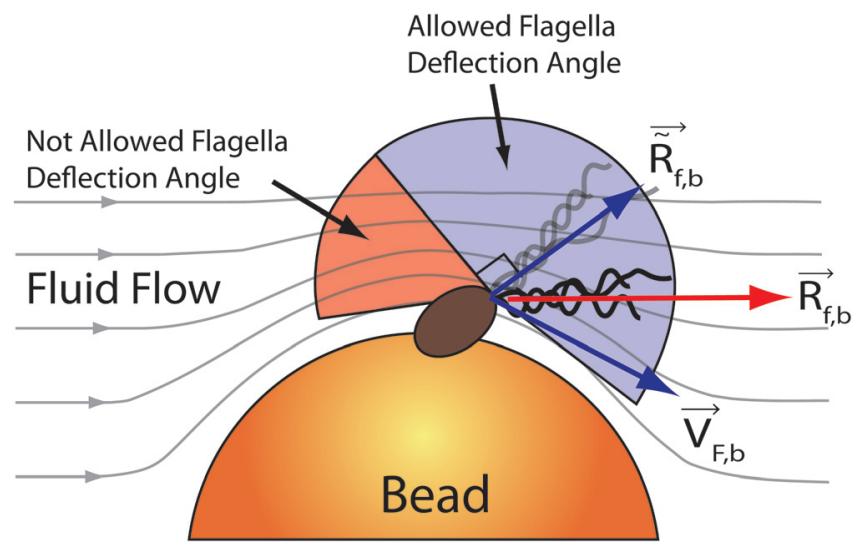

FIG. 3. (Color online) Schematic representing the concept of flexibility of the flagellar hook. The flow field around the bead will cause the flagella to deflect and align with the flow. Deflection is proportional to the flexibility of the hook. Each bacterium is assumed to have a limited range of allowable deflection angles, which, if exceeded, is assumed to induce a forced tumbling state, resulting in loss of propulsive power.

requires the consideration of the pressure field in the fluid, we instead use an alternative measure of "flexibility" of the flagellar hook. Since the amount of deflection of the flagella must be determined by the magnitude of the fluid flow around it, we define a new property for each bacterium, titled "nominal velocity," $v_{n}$. Subsequently, the orientation of each flexible flagellum during each iteration of the integration is determined by the equation:

$$
\vec{R}_{f, b}=\left(1-\frac{\left\|\vec{V}_{F, b}\right\|}{v_{n}}\right) \overrightarrow{\tilde{R}}_{f, b}+\frac{\left\|\vec{V}_{F, b}\right\|}{v_{n}} \vec{V}_{F, b}
$$

where $\vec{R}_{f, b}$ is the flagella orientation vector for bacteria $b$, $\vec{V}_{F, b}$ is the fluid flow vector at bacteria $b$, and $\overrightarrow{\vec{R}}_{f, b}$ is the flagella orientation vector if the hook were fully rigid. Therefore, the ratio $\left\|\vec{V}_{F, b}\right\| / v_{n}$ represents how much the flagella aligns with the flow and is directly related to the flexibility of the hook, being 1 for a fully flexible hook and 0 for a rigid one, see Fig. 3. This parameter, labeled "Flagella flow orientation ratio," provides intuitive meaning to the flexibility of the flagella and is used in some subsequent plots. The units of $v_{n}$ are $\mu \mathrm{m} / \mathrm{s}$, however since we are using the parameter as an effective flagellar hook stiffness indicator, its units are omitted from all subsequent discussions to reduce confusion.

Because the case of a flexible flagella hook implies a variety of possible bacteria orientation and position scenarios, some heuristics were applied to ensure physical realism of the flagella deflection. Since fluid streamlines may have a component pointing into the sphere, for example in the vicinity of the frontal stagnation point, a manual check in the simulation ensures that the bacteria can at most lie in a plane tangential to the bead at their attachment point. Furthermore, due to the original orientation randomization of bacteria, inevitably during the simulation, some bacteria will rotate such that their flagella point opposite to the flow direction and orienting with the flow will cause the flagella to lose their propulsive force. This intuition emerges from the idea that flagella, when bent $90^{\circ}$ or more, will interfere with the body of the bacteria and therefore will not be able to produce propulsive force. Extending this idea, a heuristic regarding flow orientation for flexible flagella was developed: if the fluid flow around the bead causes a bacterium's flagella to deflect more than $90^{\circ}$, a state when the flagella are producing zero propulsive force is induced in that bacterium. Hence, each bacterium has only a limited range of allowable flagella deflection angles, before wondering to an angle that would induce an off state, this concept is illustrated in Fig. 3.

\section{Fluid flow modeling}

The low Reynolds number regime in which the bacteria operate allows Stokes flow analysis to be applied when considering the fluid flow induced by the bead's motion. Since the bead will experience both translational and rotational motion, the fluid velocity in the vicinity of the bead is modeled as the superposition of linear and rotational components as portrayed in Fig. 4. The following equations were employed in modeling the flow around a translating and rotating bead:

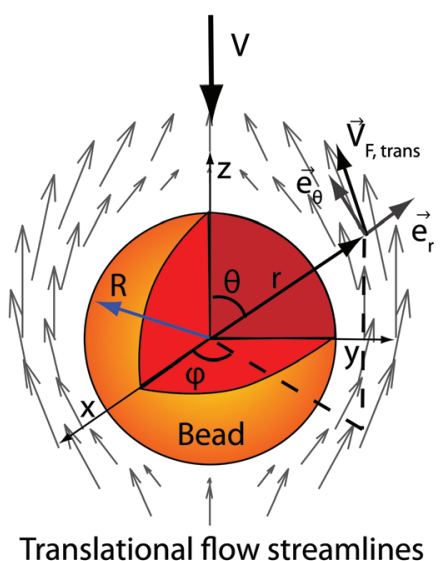

Translational flow streamlines

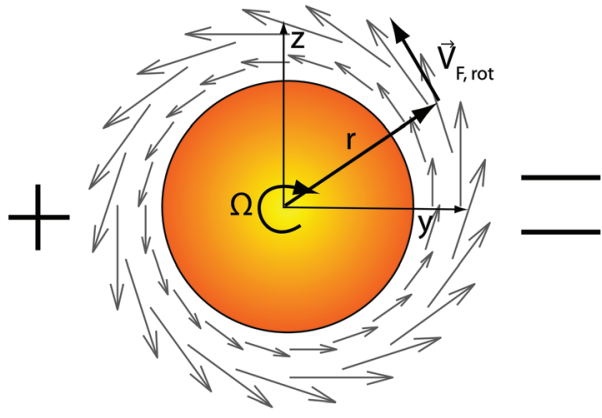

Rotational flow streamlines

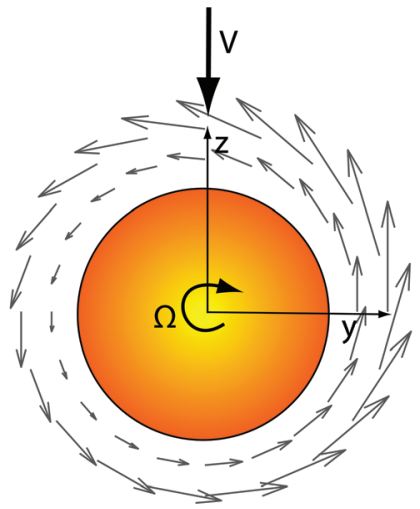

Superposition of streamlines

FIG. 4. (Color online) Schematic illustrating Stokes flow superposition to obtain the fluid velocity field around a translating and rotating spherical bead. Note that the fluid streamlines are portrayed in the body reference frame. 


$$
\begin{gathered}
\vec{V}_{F, \text { trans }}(\vec{r}, \vec{V})=\left[\|\vec{V}\| \cos \theta\left(1-\frac{3 R}{2 r}+\frac{R^{3}}{2 r^{3}}\right)\right] \vec{e}_{r} \\
+\left[-\|\vec{V}\| \sin \theta\left(1-\frac{3 R}{4 r}-\frac{R^{3}}{4 r^{3}}\right)\right] \vec{e}_{\theta}, \\
\vec{V}_{F, \text { rot }}(\vec{r}, \vec{\Omega})=\|\vec{\Omega}\| R\left(1-\frac{R^{2}}{r^{2}}\right) \vec{e}_{r} \times \vec{e}_{x}, \\
\vec{V}_{F, b}\left(\vec{r}_{b}\right)=\vec{V}_{F, \text { trans }}\left(\vec{r}_{b}, \vec{V}\right)+\vec{V}_{F, \text { rot }}\left(\vec{r}_{b}, \vec{\Omega}\right),
\end{gathered}
$$

where $\vec{V}_{F \text {,trans }}$ and $\vec{V}_{F \text {,rot }}$ represent the fluid velocity vectors for a translating and a rotating bead, respectively. The form of these equations was adapted to 3D by applying flow axial symmetry for the translational case. ${ }^{29,30}$ Furthermore, $\vec{V}$ and $\vec{\Omega}$ are the instantaneous translational and rotational bead velocity vectors, respectively, $R$ is the bead radius, and $\vec{r}$ defines the polar coordinates of the point of interest. The total velocity vector $\vec{V}_{F, b}$ at bacterium $b$ location, defined as $\vec{r}_{b}$, is calculated via superposition. In particular, as the bacterium body is $\sim 1 \mu \mathrm{m}$ in length, only the fluid velocity $1 \mu \mathrm{m}$ offset from the surface of the bead was used in the simulation.

\section{Bead dynamics}

Bacteria are modeled as point propulsive forces, having arbitrary initial orientation and state. During the tumble state, the bacteria do not produce any propulsive force, while the run state yields a constant force of $F_{b}=0.48 p N$ from each bacterium. ${ }^{13}$ Due to the Stokes flow regime, the bead's inertial effects can be ignored and hence its dynamics are governed by the equalities:

$$
\begin{gathered}
\vec{F}_{D}=6 \pi \eta R \vec{V}=-\sum_{b} F_{b} \vec{R}_{f, b} s_{b}, \\
\vec{M}_{D}=8 \pi \eta R^{3} \vec{\Omega}=-\sum_{b} \vec{r}_{b} \times F_{b} \vec{R}_{f, b} s_{b},
\end{gathered}
$$

where $\vec{F}_{D}$ is the hydrodynamic translational drag on the bead, $\vec{M}_{D}$ is the rotational drag, $\eta$ is the dynamic viscosity of the aqueous medium, $R$ is the bead radius, and $s_{b}$ is a binary variable for each bacterium, being 1 during the run phase and 0 for the tumble phase. During each integration step $\mathrm{dt}=0.05 \mathrm{~s}$, the resultant linear and angular velocities of each bead, emerging from the contribution of all bacteria attached to it, are evaluated and its motion is simulated forward in time. After each iteration, a routine check is performed to ensure that no bacterium's flagella is deflecting more than $90^{\circ}$, in which case an "off" state is induced for that bacterium for the next time iteration. Random Brownian linear and angular displacements have been also included in the numerical model by allowing random displacement and rotation of the beads during each iteration characterized by root mean squared linear displacements of $\sqrt{2 D d t}$ in each of the three $x, y, z$ directions, and angular displacements of $\sqrt{2 D_{R} d t}$ around a random axis, where $D$ and $D_{R}$ are defined after Eq. (8). However the displacements caused by these processes are orders of magnitude less than those caused by bacteria propulsion and therefore are unnoticeable.

\section{SIMULATION RESULTS AND DISCUSSION}

The theoretical simulation presented above is validated against the experimental work previously reported by Behkam and Sitti. ${ }^{21}$ Since the motion of the beads is a random walk, it is fundamentally impossible to directly compare the experimental and theoretical bead trajectories. Instead, as basis for comparison, the mean velocity $V_{\text {mean }}$ acquired by the beads when propelled by bacteria is used. In general, the mean squared displacement of microbeads subjected to stochastic translation and rotation in 3D can be described by the equation (adapted from Refs. 31 and 32):

$$
\Delta L^{2}=6 D \Delta t+\frac{V_{\text {mean }}^{2} \tau_{R}^{2}}{2}\left[\frac{2 \Delta t}{\tau_{R}}+e^{-2 \Delta t / \tau_{R}}-1\right],
$$

where $V_{\text {mean }}$ is the bead velocity magnitude averaged over its trajectory [note that this is different from $\vec{V}$ which is the bead's instantaneous linear velocity from Eq. (6)], $D$ is the diffusion coefficient (defined as $k_{B} T / 6 \pi \eta R$, where $k_{B}$ is the Boltzmann constant and $T$ is the absolute temperature), $\tau_{R}$ is the rotational diffusion randomization time $\left(\tau_{R}^{-1}=D_{R}=\right.$ $k_{B} T / 8 \pi \eta R^{3}$ ), which is $\tau_{R} \approx 11 \mathrm{~min}$ for $10 \mu \mathrm{m}$ beads, and $\Delta t$ is the simulation time. ${ }^{21}$ Since, experimental runs range for 6 $\mathrm{s}$, which is much less than the randomization time, we can express the exponential term by its Taylor approximation in the vicinity of 0 and Eq. (8) simplifies to:

$$
\Delta L^{2} \approx 6 D \Delta t+V_{\text {mean }}^{2} \Delta t^{2} .
$$

Furthermore, given that the effect of bacteria propulsion is much greater than diffusion, shown by experimental data $\mathrm{in}^{21}$ which serves as the basis for this work, Eq. (9) simplifies to its final form used in this work $\Delta L^{2} \approx V_{\text {mean }}^{2} \Delta t^{2}$. The values of $V_{\text {mean }}$ in all the results presented below were obtained by fitting the simulated squared displacement trajectories to the above equation in the least squared error sense.

\section{A. Determination of flagellar hook flexibility}

Since varying the flexibility of the flagellar hook significantly alters the dynamics and therefore the trajectories of the beads, characterization of this property is attempted via comparison to experimental results, as well as, to statistical theory. Mean bead velocities resulting from varying the hook flexibility are superposed with experimental results of Behkam et al., ${ }^{21}$ and the velocity trends produced by increasing the bead size are compared to statistical arguments of Darnton et al. ${ }^{13}$

\section{Velocity sensitivity to flagellar hook flexibility}

Patterned and unpatterned bead motion is simulated and the sensitivity of obtained bead mean velocities to flexibility of the hook is portrayed in Fig. 5. The primary purpose of this plot is to establish a hook flexibility, encoded in the parameter $v_{n}$ of Eq. (2), that agrees best with experiments. Overall the mean velocities obtained from simulation are higher than the experimentally obtained values of $V_{u}^{*}=14.8 \mu \mathrm{m} / \mathrm{s}$ for unpatterned beads, $V_{p}^{*} \approx 18 \mu \mathrm{m} / \mathrm{s}$ for patterned beads with 10 bacteria attached, and $V_{p}^{*} \approx 21 \mu \mathrm{m} / \mathrm{s}$ for 13 bacteria attached 


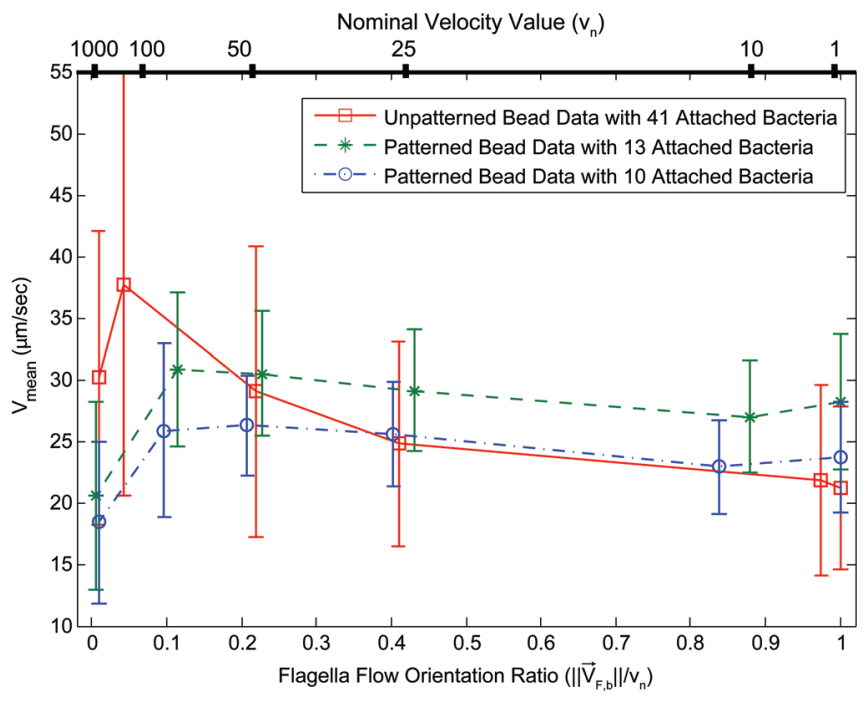

FIG. 5. (Color online) Simulated sensitivity of bead mean velocity to the flexibility of the flagellar hook. The velocities have been averaged over the motion of 100 beads for each run and the values of hook flexibility have been averaged overall attached bacteria. Unpatterned beads featured 41 bacteria uniformly spaced over the entire bead surface, while patterned beads featured 10 and 13 bacteria in close packed hexagonal configuration clustered together. The bacterial hook flexibility is conveyed by dual axes, "Nominal Velocity" (top $x$ axis) and the "Flagella Flow Orientation Ratio" (bottom $x$ axis) portraying the relationship between those quantities.

(where the asterisk denotes experimental results). This could potentially be attributed to the hydrodynamic interactions between neighboring bacteria reducing their individual propulsive force, effect not accounted for in the simulation where the bacteria are modeled as independent entities. Although no quantitative conclusions can be drawn, experimental data presented in Appendix A suggests that separation distance between neighboring bacteria adversely affects their propulsive force. Indeed, overall propulsive force reduction would shift all simulated velocity curves down, yielding better agreement with experimental results. Given the fact that the velocity behavior for all simulated cases is nonmonotonic and features a wide range of acceptable hook flexibility values, the determination of this parameter based on this plot alone would result in a very uncertain value. Hence, other simulation means are employed to shed more light on the problem.

\section{The effect of bead radius on the motion}

Using statistical methods accounting for the bacterial distribution on an object combined with their run/tumble behavior, one can determine that the overall propulsive force will scale as $F \propto L$, where $L$ is the length scale of the object. Since the hydrodynamic drag on the object also scales with $L$, it can be concluded that object's speed will be independent of the object's size. ${ }^{13}$ This concept is used as means to characterize bacterial hook flexibility. Since the size of the bead also alters the magnitude of the fluid flow in the bead's vicinity, the metric of Flexibility of the Hook ranging between 0 and 1 becomes inadequate, as this number changes with the bead radius. The metric of choice then is the value of "nominal velocity" $v_{n}$, defined in Eq. (2), as it provides a more direct measurement of the physical stiffness of the flagellar hook. Furthermore, results of simulations por- traying the variation of unpatterned and patterned bead velocities to the bead radius and flagellar hook flexibility are portrayed in Fig. 6. Unlike in previous results, where bacteria were attached to the beads with uniform spacing, in these simulations the bacteria attachment points were sampled from a uniform distribution, as this is the most general scenario of attachment since little information is known about attachment, due to the lack of experiments performed for such large beads.

It is apparent that unpatterned beads exhibit much greater sensitivity to bacterial hook flexibility than the patterned ones. The explanation of this behavior comes from the nature of fluid flow around the unpatterned beads. Generally, due to the configuration and alignment of bacteria on the surface of the bead, rotational motion is favored over translational, thus generating rotational fluid flow in the vicinity of the bead's surface. In the case of a very flexible

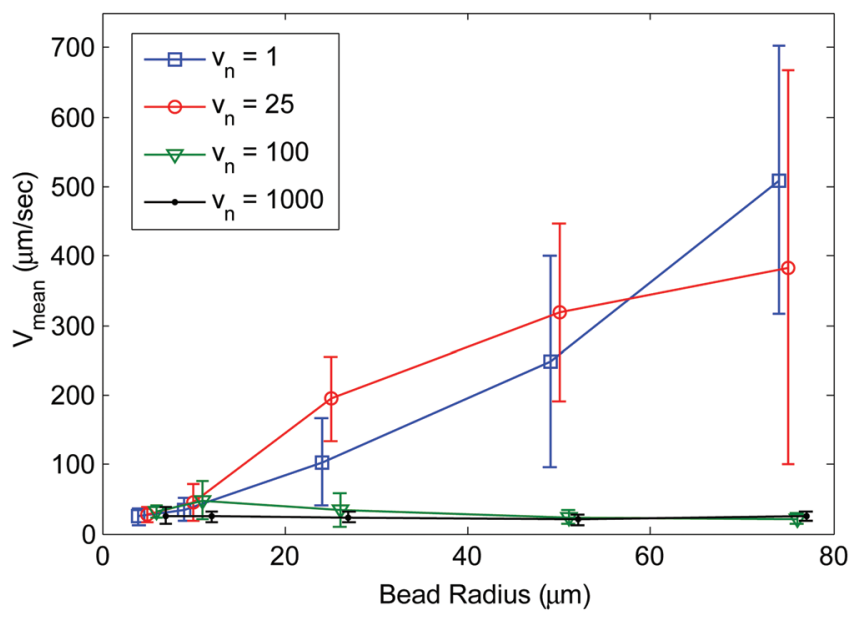

(a)

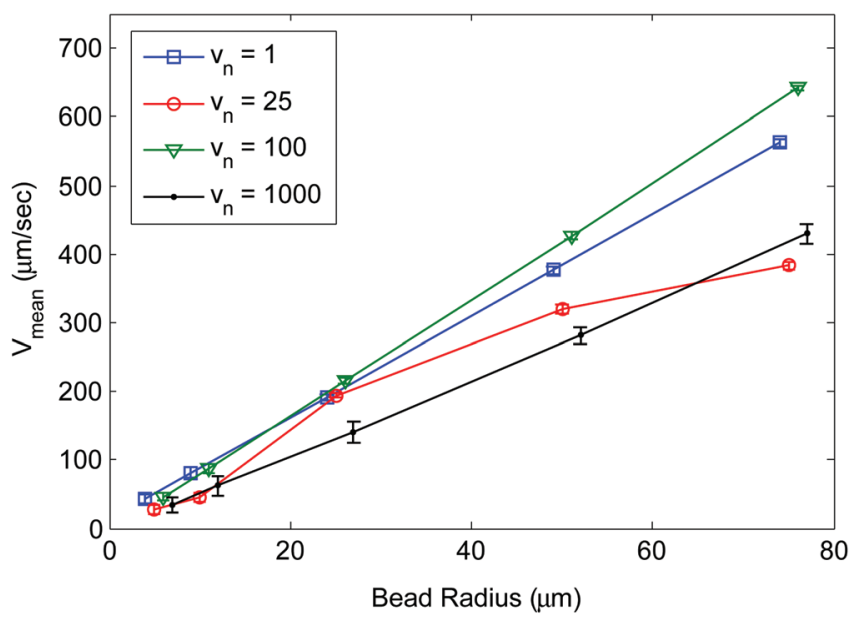

(b)

FIG. 6. (Color online) Variation of bead velocities with bacterial hook flexibility and bead radius for (a) unpatterned and (b) patterned beads. A set of four nominal velocity values was considered in all cases $v_{n}=\{1,25,100,1000\}$, where 1 denotes a very flexible flagella and 1000 denotes an almost rigid one. All simulations were performed for 10 beads. Note that the radii of the beads were offset from their actual values in the plot for clarity. Unpatterned beads featured randomly attached bacteria whose number was computed by considering that bacteria generally attach with density of 1 bacterium per $7 \mu \mathrm{m}^{2}$, while for patterned beads the assumed bacterial density was 1 bacterium per $12 \mu \mathrm{m}^{2}$. 
flagellar hook, flagella will easily orient in the direction of rotational flow, thus amplifying the moment on the bead and increasing the angular velocity even more. Furthermore, given that bacteria attach with a uniform density to the surface of the bead, their number $N$ is proportional to the bead radius $R, N \propto R^{2}$, and their overall produced force $F \propto \sqrt{N} \propto R$, hence the total rotational moment is $M \propto R \times F \propto R^{2}$. Furthermore, since resulting angular velocity of a sphere is a balance between moment and hydrodynamic rotational drag [Eq. (7)], $\Omega \propto M / R^{3} \propto R^{-1}$, thus the angular velocity decays as the inverse of radius of the beads. Therefore when the angular component of velocity decreases for larger beads, the translational flow component begins to dominate, and the aligned to it flagella will propel the bead linearly, thus achieving large speeds. On the other hand, rigid flagella are independent of the fluid flow around them, hence the above described effect does not pertain to them. Indeed, simulation results convey this behavior as the trajectories of large beads propelled by flexible flagella are long with minimal direction change, as portrayed in Fig. 7(a), while the rigid flagella case exhibits much shorter trails with a lot of direction changes, Fig. 7(b). Thus, the case of rigid flagella, showing virtually no sensitivity of bead speed to its radius, agrees best with theoretical considerations, presented in the beginning of this section. Although, the unavailability of actual experimental data for large bead sizes for comparison precludes any definite conclusions, the presented results suggest a somewhat stiff flagellar hook.

\section{Sensitivity of velocity values of patterned beads to number of attached bacteria}

The effect of flagellar hook stiffness is also investigated in the context of a small number of bacteria attached to patterned beads and compared with experimental results of. ${ }^{21}$ Due to unknown hydrodynamic, bacterial behavior and attachment effects, the simulation does poorly on predicting bead velocities propelled by less than eight bacteria, hence modeling of only eight or more attached bacteria on patterned beads is performed. As shown in Fig. 8, flexibility of the flagella has a significant impact on bead velocities, with the best fit to experimental results being the case of $v_{n}=1000$, representing a very stiff flagellar hook. Due to better agreement of predicted mean velocity values with experimental results, these simulations suggest a stiff flagellar hook.

Throughout the presented simulation scenarios, flexibility of the bacterial flagellar hook is shown to play a major role in determining the mean velocity as well as the overall shape of bead trajectories. Two scenarios, namely, the sensitivity of bead velocity to bead size and to the number of attached bacteria for patterned beads, yielded much better agreement to experiment and theoretical considerations in the case of a stiff flagellar hook. However, mean velocities obtained for unpatterned beads for the same case of high hook stiffness are overestimated $(30 \pm 12 \mu \mathrm{m} / \mathrm{s}$ in simulation versus $14.8 \pm 1.1 \mu \mathrm{m} / \mathrm{s}$ in experiment), as portrayed in Fig. 5. Given the fact the simulation predicts much better mean velocities for the patterned beads $(18.4 \mu \mathrm{m} / \mathrm{s}$ and $21.6 \mu \mathrm{m} / \mathrm{s}$

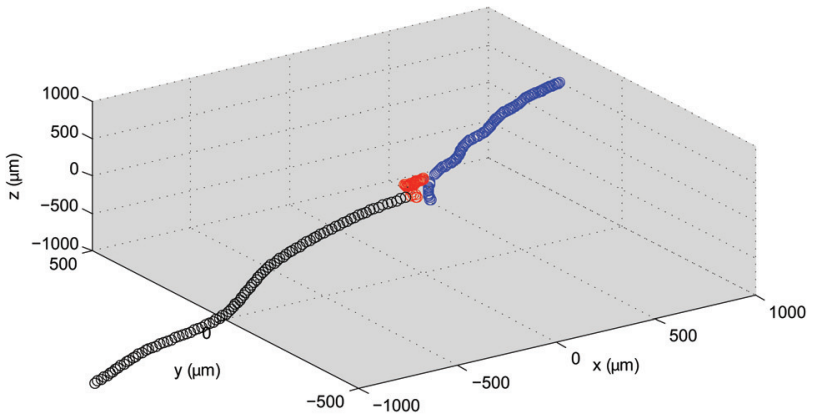

(a)

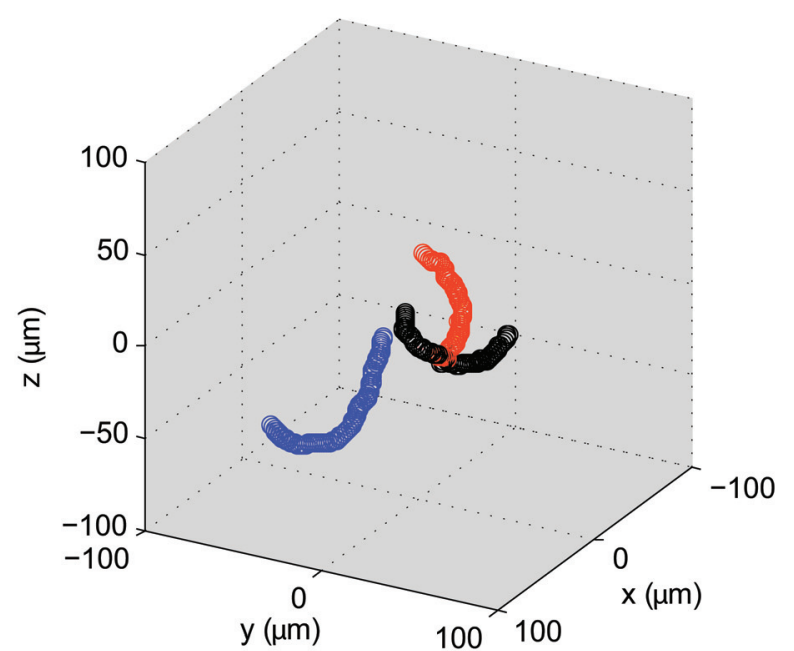

(b)

FIG. 7. (Color online) Simulated 3D trajectories of 3 unpatterned beads: a) bacteria with $v_{n}=1$ attached to a $50 \mu \mathrm{m}$ radius bead, and (b) bacteria with $v_{n}=1000$ attached to a $75 \mu \mathrm{m}$ bead. Simulations feature randomly distributed attached bacteria whose number was computed by considering that bacteria generally attach with density of 1 bacterium per $7 \mu \mathrm{m}^{2}$.

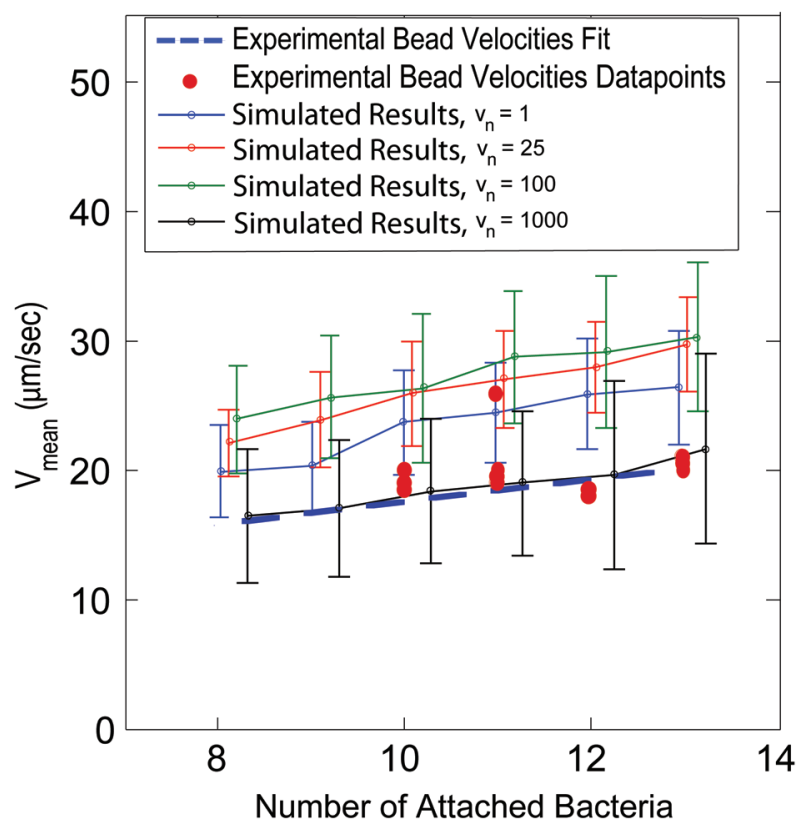

FIG. 8. (Color online) Sensitivity of the bead mean velocity to the number of attached bacteria and flagellar hook flexibility for patterned beads. The velocities have been averaged over the motion of 100 beads for each run. 


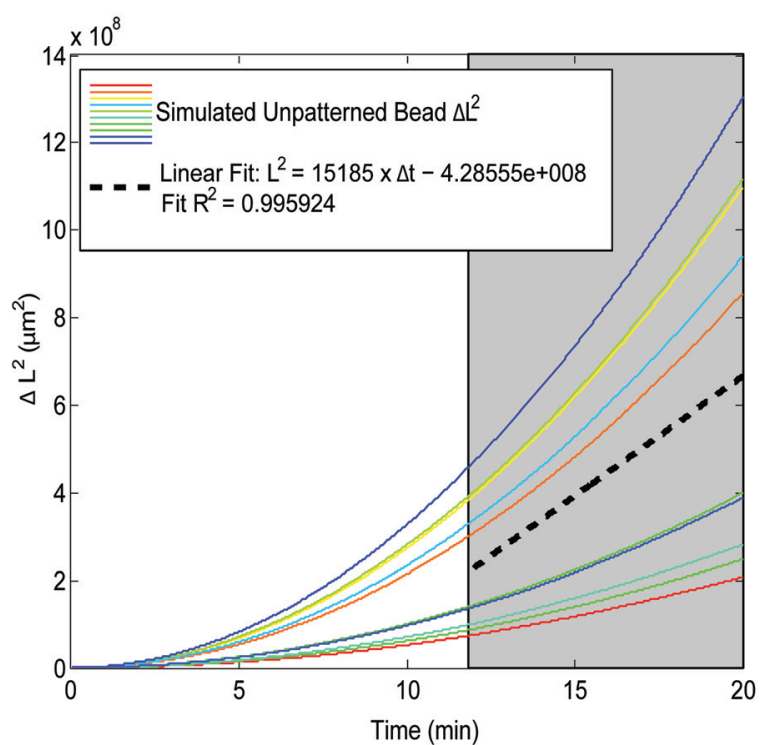

(a)

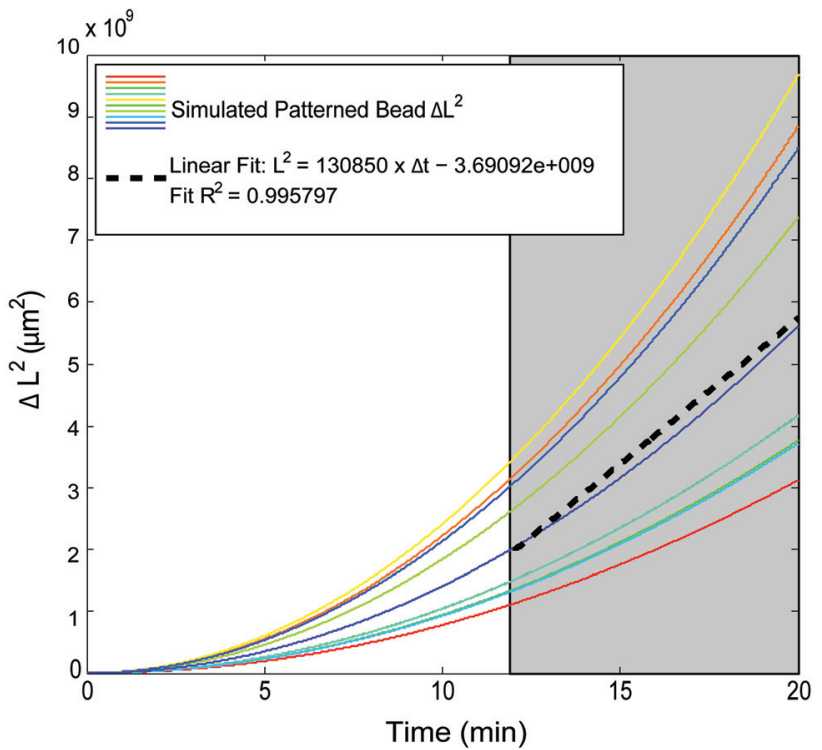

(b)

FIG. 9. (Color online) Random walk behavior of unpatterned (a) and patterned (b) $20 \mu \mathrm{m}$ diameter beads. The simulations were performed for a stiff flagellar hook with $v_{n}=1000$. Simulation was run for a time range of $\Delta t=[0-20] \min \left(\Delta t \gg \tau_{R}\right)$ for 10 beads. The gray area represents the "random walk" regime, encompassing the time period [12-20] min. The shown regression fit is performed overall 10 trajectories of bead motion for both unpatterned and patterned cases.

in simulation versus $18 \mu \mathrm{m} / \mathrm{s}$ and $21 \mu \mathrm{m} / \mathrm{s}$ in experiment, for 10 and 13 attached bacteria, respectively) and that the experimentally measured bacteria attachment density is almost twice higher in unpatterned than patterned beads, the discrepancy for the unpatterned bead prediction is attributed to a greater level of hydrodynamic interaction between neighboring flagella, thus reducing propulsion. However, given the highly stochastic nature of the system and the lack of experimental data for all simulated scenarios, such as velocities for larger beads and interaction between flagella on spherical geometry, the exact stiffness of the bacterial flagellar hook cannot be determined, but only inferred to behave more as a rigid flagella than a flexible one. Furthermore, given that bacteria produce propulsive force by bundling multiple flagella together, another interpretation of the above result is that the flagella bundle might be stiff, for example due to its assembly geometry, hence presenting no information about the flexibility of the hook of individual flagella.

\section{B. Random walk behavior}

To employ the theoretical model as a simulation tool, we simulate the behavior of the beads after the randomization time has been exceeded, an experiment that could not be performed in reality due to equipment limitations. Given that the above discussion reinforced the idea that the flagellar hook acts as a stiff entity, the long time bead behavior has been simulated with a nominal velocity value of $v_{n}=1000$. Reverting to Eq. (8), for the case of $\Delta t \gg \tau_{R}$ it can be simplified to:

$$
\Delta L^{2} \approx\left(6 D+V_{\text {mean }}^{2} \tau_{R}\right) \Delta t=D_{\text {eff }} \Delta t
$$

where $D_{\text {eff }}$ is the effective diffusion coefficient of the beads. Figure 9 presents the $\Delta L^{2}$ trajectories for 20 min simulation runs of patterned and unpatterned $20 \mu \mathrm{m}$ diameter beads.
The linear behavior for $\Delta t>11 \mathrm{~min}$ is indeed observed as expected; hence, we fit the trajectories in the time domain of $12-20 \mathrm{~min}$ to Eq. (10). The effective diffusion coefficient for the patterned $20 \mu \mathrm{m}$ diameter beads $\left(D_{\text {eff }, p}\right.$ $=130850 \mu \mathrm{m}^{2} / \mathrm{min}$ ) is an order of magnitude greater that of the unpatterned beads $\left(D_{\text {eff }, u}=15185 \mu \mathrm{m}^{2} / \mathrm{min}\right)$ reinforcing the idea that patterned beads do indeed produce more directed propulsion at enhanced velocities with a significantly smaller number of attached bacteria.

\section{CONCLUSION}

In summary, a stochastic model for bacteria propulsion of microbeads is developed. The model captures the effect of key factors such as orientation and attachment configuration of bacteria onto the bead, run and tumble rates, and the flexibility of the flagellar hook. The emergent numerical simulation was employed to shed light on the stiffness of the flagellar hook of the bacteria via the examination and comparison of several simulation results to experimental data. Having examined the sensitivity of mean bead velocity to the size of the bead and the number of attached bacteria, the results suggest a more stiff flagellar hook rather than a flexible one. Using this obtained stiffness value the numerical model was also used as a prediction tool enabling simulation of experiments not yet performed in reality, such bead motion for times much greater than the randomization time. Furthermore, the simulation presented here can easily be expanded to encompass chemotactic behavior of the bacteria in chemical gradients. Also, although the simulation results are presented for spheres with a small number of attached bacteria, the model can be modified for other geometries fairly easily, by redefining bacteria attachment rules and hydrodynamic effects around the new geometry. These investigations require more experimentation on the 
configuration of the bacteria attachment for more complex shapes, and are seen as future works.

As a shortcoming of the developed model, it was not able to accurately capture bead dynamics in a few cases, most notably the overprediction of unpatterned bead velocities when propelled by very stiff bacterial flagella. This discrepancy can be potentially attributed to the hydrodynamic interactions between bacteria in a close packed configuration. Although some scaled up experiments suggesting the effect of propulsive force reduction between neighboring bacteria are outlined in Appendix A, more experimental data on spherical geometry is required for identification and characterization of these hydrodynamic effects and their implementation in the theoretical model. Future experimental insight into these effects coupled with a theoretical stochastic propulsion model, alike presented in this work, will potentially enable design and control of bacteria propelled swimming microrobots for future targeted drug delivery applications in stagnant or low velocity body fluids.

\section{ACKNOWLEDGMENTS}

The authors thank Ramin Golestanian for insightful discussions in development of mathematical models. Experimental measurements for scaled up flagella experiments were obtained with the aid of Akshay Jayaram and Umit Danis. This work was partially supported by National Science Foundation (IIS-0713354).

\section{APPENDIX A: FLUIDIC COUPLING INTERACTION BETWEEN NEIGHBORING FLAGELLA VIA SCALED- UP EXPERIMENTS}

To examine the coupled fluidic interaction between neighboring flagella, the thrust force produced by multiple flagella at low Reynolds number was measured experimentally. The physical dimensions of the flagella were scaled up and tested in a silicone oil 30000 times more viscous than water, reducing the Reynolds number of the experiment to $\approx 0.007$, and ensuring similar fluid dynamics between the real and scaled up flagella. A photograph of the experimental measurement setup is shown in Fig. 10.

In order to achieve individual rotation of multiple flagella with variable spacing between each other, a planetary gear system was fabricated. The central sun gear was driven by a small DC motor with a built-in 30:1 gear box (COPAL 30:1 Gearmotor) intended for high torque. Three planet gears in a stationary carrier plate were evenly spaced around the sun gear. Additional planet gears to which flagella could be coupled were placed radially outward to obtain four different spacings $(9.4,13.3,17.1$, and $21.0 \mathrm{~mm}$ ) between flagella when attached equidistant to the central sun gear. Each flagellum was fabricated by bending $0.4 \mathrm{~mm}$ diameter steel wire into a helix (4 mm diameter, $6 \mathrm{~mm}$ pitch, $30 \mathrm{~mm}$ length) with a straight axial leader for coupling (via a Delrin coupler) to the planet gear. Only the flagella and leader were submerged in the silicone oil, requiring the leader to be long enough to limit effects from the oil surface. Additionally, the flagella were placed in the center of the large ( 8 by 16 in.) tank of oil to limit wall effects. The base of the motor was

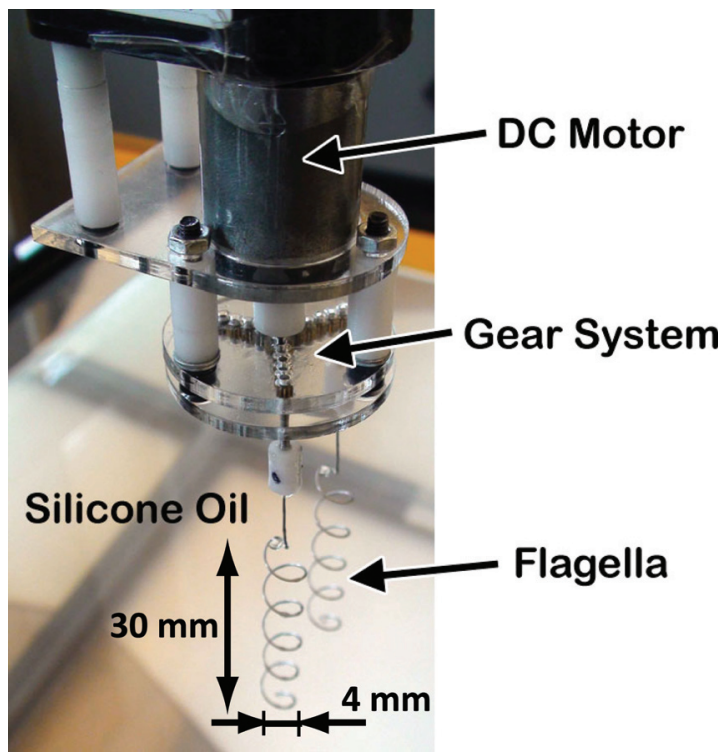

FIG. 10. (Color online) Photograph of the setup for measuring the thrust force produced by multiple flagella. The planetary gear system was used to guarantee individual rotation of each flagella at identical rates.

attached directly to the load point of a $50 \mathrm{~g}$ load cell (Transducer Techniques GSO-50), and the force readings were recorded on a computer through a data acquisition board for subsequent analysis. The rotational speeds of the flagella were controlled by manually adjusting the voltage supplied to the motor, with a strobe light used to measure the rotation. Measurements were made for 1, 2, and 3 flagella at a range of rotational frequencies from 1.5 to $4 \mathrm{~Hz}$, in intervals of 0.5 $\mathrm{Hz}$, as well as 4 different spacings between neighboring flagella. Each measurement was performed three times.

The results of the thrust force measurements are plotted in Fig. 11(a). First, the three flagella were tested individually, ensuring that fabrications differences were minor. Evidence of fluidic interaction between neighboring flagella was apparent. Visually, the flagella could be seen to bend toward each other, with the effect increasing with higher rotational speeds. Indeed, 3 flagella at the highest frequency and closest spacing resulted in physical entanglement after a short period of time, and the plotted thrust force corresponds to the highest measurement prior to entanglement.

In order to better investigate the effect of interaction between neighboring flagella on the overall thrust force, the multiple flagella data was normalized by dividing the measured force by the average single flagella force for each rotational frequency (plotted in Fig. 11(b)). Assuming no interaction, one would expect the normalized thrust force to be equal to the number of flagella, yet from the results, it is clear that neighboring flagella are negatively affecting the thrust force. Furthermore, the data shows that both greater rotational frequency and closer spacing increases the effect. These results make intuitive sense when one considers the fluid flow around the helices. The rotation of a single helix causes the viscous fluid to rotate with it. A second helix in the same fluid will do the same, but if the helices are rotating in the same direction, the fluid in between is pulled in different directions. Energy that might otherwise be put toward propulsion is instead spent on resolving these conflicting 


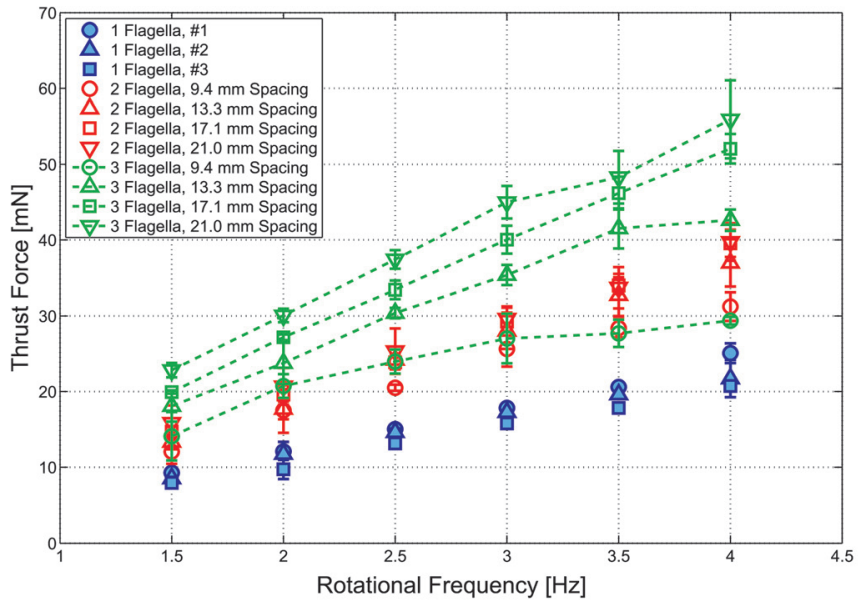

(a)

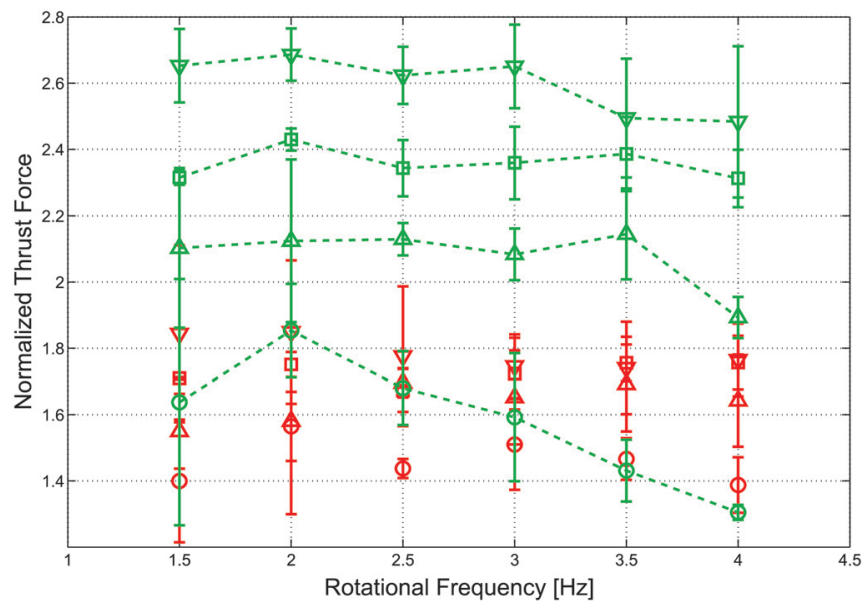

(b)

FIG. 11. (Color online) Thrust force measurement results: (a) Data plotted as measured thrust force versus rotational frequency. Single flagella measurements are filled markers, two flagella measurements are open markers, and three flagella measurements are open markers connected with a dashed line. (b) Same data plotted as normalized thrust force vs rotational frequency. The multiple flagella measurements were divided by the average single flagella force for each rotational frequency. Measurement statistics were obtained from three measurements for each case.

forces. Although the collected data clearly demonstrates the presence of fluid interaction between neighboring flagella and its detrimental impact on the overall produced force, it does not fully characterize the phenomenon, since the effect of flagellar helix pitch and diameter are future works.
${ }^{1}$ M. Sitti, Nature 458, 1121 (2009).

${ }^{2}$ B. R. Donald, C. G. Levey, C. D. McGray, I. Paprotny, and D. Russ, J. Microelectromech. Syst. 15, 1 (2006).

${ }^{3}$ C. Pawashe, S. Floyd, and M. Sitti, Int. J. Robot. Res. 28, 1077 (2009).

${ }^{4}$ K. B. Yesin, K. Vollmers, and B. J. Nelson, Int. J. Robot. Res. 25, 527 (2006).

${ }^{5}$ R. Dreyfus, J. Baudry, M. L. Roper, M. Fermigier, H. A. Stone, and J. Bibette, Nature 437, 862 (2005).

${ }^{6}$ L. Zhang, J. J. Abbott, L. X. Dong, B. E. Kratochvil, D. J. Bell, and B. J. Nelson, Appl. Phys. Lett. 94, 064107 (2009).

${ }^{7}$ R. K. Soong, G. D. Bachand, H. P. Neves, A. G. Olkhovets, H. G. Craighead, and C. D. Montemagno, Science 290, 1555 (2000).

${ }^{8}$ H. Berg, Ann. Rev. Biochem. 72, 19 (2003).

${ }^{9}$ S. H. Larsen, J. Adler, J. J. Gargus, and R. W. Hogg, Proc. Natl. Acad. Sci. U.S.A. 71, 1239 (1974).

${ }^{10}$ S. Kojima and D. F. Blair, Int. Rev. Cytol. 233, 93 (2004).

${ }^{11}$ L. L. McCarter, J. Mol. Microbiol. Biotechnol. 7, 18 (2004).

${ }^{12}$ E. Leifson, Atlas of Bacterial Flagellation (Academic, New York, 1960).

${ }^{13}$ N. Darnton, L. Turner, K. Breuer, and H. C. Berg, Biophys. J. 86, 1863 (2004).

${ }^{14}$ M. Kim and K. Breuer, J. Fluids Eng. 129, 319 (2007).

${ }^{15}$ E. Steager, C. Kim, J. Patel, S. Bith, C. Naik, L. Reber, and M. Kim, Appl. Phys. Lett. 90, 263901 (2007).

${ }^{16}$ S. Tung and J. Kim, IEEE Int. Conf. Robot. Biomimetics, (China, 2004) p. $21-25$.

${ }^{17}$ S. Martel, C. Tremblay, S. Ngakeng, and G. Langlois, Appl. Phys. Lett. 89, 233904 (2006).

${ }^{18}$ S. Martel, W. Andr, M. Mohammadi, and Z. Lu, "Towards swarms of communication-enable and intelligent sensotaxis-based bacterial microrobots capable of collective tasks in an aqueous medium," IEEE International Conference on Robotics and Automation (IEEE, Kobe, Japan 2009).

${ }^{19}$ B. Behkam and M. Sitti, Appl. Phys. Lett. 90, 023902 (2007).

${ }^{20}$ B. Behkam and M. Sitti, "Bacterial propulsion of chemically patterned micro-cylinders," Proceedings of the IEEE-RAS-EMBC International Conference on Biomedical Robotics and Biomechatronic (IEEE, Scottsdale, AZ, 2008), pp. 753-757.

${ }^{21}$ B. Behkam and M. Sitti, Appl. Phys. Lett. 93, 223901 (2008).

${ }^{22}$ B. Behkam and M. Sitti, "Characterization of bacterial actuation of microobjects," Proceedings of IEEE Conference on Robotics and Automation (ICRA), (IEEE, Kobe, Japan, 2009), pp. 1022-1027.

${ }^{23}$ A. A. Julius, M. S. Sakar, E. Steager, U. K. Cheang, M. Kim, V. Kumar, and G. J. Pappas, "Harnessing bacterial power for micro scale manipulation and locomotion," IEEE International Conference on Robotics and Automation (IEEE, Kobe, Japan, 2009).

${ }^{24}$ S. M. Block, J. E. Segall, and H. C. Berg, Cell 31, 215 (1982).

${ }^{25} \mathrm{H}$. Berg, E. coli in Motion (Springer, Berlin, 2004).

${ }^{26}$ H. Berg, Phys. Today 53, 24 (2000).

${ }^{27}$ S. M. Block, D. F. Flair, and H. C. Berg, Nature 338, 514 (1989).

${ }^{28}$ S. M. Block, D. F. Blair, and H. C. Berg, Cytometry 12, 492 (1991).

${ }^{29}$ J. Happel and H. Brenner, Low Reynolds Number Hydrodynamics, 1 st ed. (Prentice-Hall, Englewood Cliffs, NJ, 1965), pp. 120-125.

${ }^{30}$ I. G. Currie, Fundamental Mechanics of Fluids, 2nd ed. (McGraw-Hill, New York, 1993), p. 260.

${ }^{31}$ J. R. Howse, R. A. L. Jones, A. J. Ryan, T. Gough, R. Vafabakhsh, and R. Golestanian, Phys. Rev. Lett. 99, 0481021 (2007).

${ }^{32}$ R. Golestanian, Phys. Rev. Lett. 102, 1883051 (2009). 